Voix et Images

volxetimages

\title{
Un guide à suivre, un répertoire à compléter
}

\section{Jacques Allard}

Volume 15, numéro 1 (43), automne 1989

Jacques Poulin

URI : https://id.erudit.org/iderudit/200825ar

DOI : https://doi.org/10.7202/200825ar

Aller au sommaire du numéro

\section{Éditeur(s)}

Université du Québec à Montréal

ISSN

0318-9201 (imprimé)

1705-933X (numérique)

Découvrir la revue

Citer cet article

Allard, J. (1989). Un guide à suivre, un répertoire à compléter. Voix et Images, 15(1), 127-131. https://doi.org/10.7202/200825ar d'utilisation que vous pouvez consulter en ligne.

https://apropos.erudit.org/fr/usagers/politique-dutilisation/ 


\section{Recherches}

\section{Un guide à suivre, un répertoire à compléter}

\section{par Jacques Allard, Université du Québec à Montréal}

Voici une nouvelle chronique que nos lecteurs trouveront dorénavant dans chacune de nos livraisons. Il s'agira pour son responsable d'informer (et d'offrir quelques commentaires) sur les travaux en cours ou à venir, d'ici et d'ailleurs, dont il aura connaissance dans le domaine des études québécoises ${ }^{1}$. Que faire pour commencer? Signaler deux publications récentes dont on n'a pas encore beaucoup parlé: un Guide de la littérature québécoise ${ }^{2}$ et Études canadiennes: publications et thèses étrangères ${ }^{3}$. Ce sont là deux ouvrages qui démontrent à leur face même ce qu'est devenue la littérature québécoise: un 
objet d'études autonome dont le rayonnement à l'étranger connait depuis quelque dix ans un certain essor.

\section{Le Guide Fortin-Lamonde-Ricard}

Signé par des chercheurs et enseignants de McGill, le Guide de la littérature québécoise deviendra vite indispensable pour tous (s'il est régulièrement mis à jour): pense-bête pour les spécialistes et pilote automatique pour tout chercheur et enseignant débutant. Il s'agit en effet d'un dispositif bien monté, portant non sur les auteurs ou les cuvres littéraires mais bien sur tous les types d'études dont on aura besoin pour démarrer ou avancer dans quelque champ que ce soit. Car, comme on le dit avec justesse en introduction: Il vise aussi à dresser un tableau de l'état actuel de la recherche, où soient reflétés aussi fidèlement que possible ses points forts et ses secteurs moins bien développés.

Après avoir présenté, dans les deux premières parties, les ouvrages de fond (dictionnaires, bibliographies, histoires littéraires, ouvrages généraux, périodes et courants, chronologies, anthologies), il renvoie ensuite aux études portant sur tous les genres, en incluant la littérature personnelle, celle de la jeunesse, celles de la radio et de la télévision ou encore la paralittérature, ce qui témoigne de l'expansion récente du corpus. Viennent ensuite quatre parties sur les «réseaux» (littérature des communautés culturelles, études comparées, la francophonie, etc); l'imprimé et la langue; la recherche; le contexte (histoire et société, champs culturels connexes), tous aspects qui montrent cette fois les ramifications nouvelles de l'objet d'études. Le Guide s'achève sur un appendice où sont regroupées les études concernant les femmes, l'institution littéraire et les écrits de la Nouvelle-France, trois orientations importantes des travaux depuis une dizaine d'années. On trouve enfin un index des noms d'auteurs ou directeurs d'ouvrages cités.

Chacune de ces parties mériterait d'être analysée. On se contentera, pour l'instant, de prendre pour exemple la cinquième, intitulée «La recherche». Trois rubriques se présentent. La première s'intitule «État de la recherche» et propose d'abord trois titres d'ouvrages-bilans: l'un de 1968 - Recherche et littérature canadienne-française de Wyczynski, Ménard et Hare -, l'autre de 1978 Situation de l'édition et de la recherche (littérature québécoise ou canadienne-française) de R. Dionne - et le numéro de Voix \& images (XII, $2,1987)$ sur les revues universitaires. L'énumération de ces titres bien connus (sans oublier les autres auxquels on est renvoyé) nous amène tout de suite à un constat: nous n'avons pas de bilan pour l'ensemble des recherches faites depuis plus de dix ans.

Qui organisera le colloque, publiera l'ouvrage de synthèse dont nous aurions besoin pour relancer le travail, guider les étudiants? Faisons-nous encore les thèses thématiques qui étaient encore si importantes jusqu'en 1982? Étudions-nous encore de préférence Anne Hébert, Jacques Ferron, Réjean Ducharme et Hubert Aquin? Est-ce que nous continuons de négliger ce qui précède 1940, en particulier les années vingt? Est-ce que les thèses et mémoires portent encore à plus de $80 \%$ sur le $\mathrm{XX}^{\mathrm{e}}$ siècle? Et les thèses dites «de 
création», ont-elles continué leur expansion (jusqu'27 \% à Sherbrooke)? Est-ce que les études comparées sont encore aussi négligées (5\%)? L'Université d'Ottawa produit-elle toujours plus de thèses que n'importe quelle université du Québec (plutôt productrices de mémoires de maîtrise)? 4

À propos des thèses et mémoires, il faudrait une suite au Répertoire Naaman qui va jusqu'en 1976. Le Guide Fortin-Lamonde-Ricard fournit déjà les sources à qui voudra bien assumer la tâche. Toujours sous cette rubrique de la «recherche», on y trouvera aussi référence au Répertoire des professeurs et chercheurs de $R$. Dionne. Il en va de même pour les revues savantes (liste de quatorze), les bibliothèques, les dépôts d'archives, les centres et équipes de recherche. Sur ces derniers, il signale que quatre groupes [...] se détachent par l'ampleur de leurs ressources et de leurs travaux: le Centre de recherche en littérature québécoise (CRELIQ) de Laval, le Corpus d'éditions critiques d'Ottawa, l'Édition critique de l'œuvre d'Hubert Aquin (ÉDAQ) à l'UQAM et le Groupe de recherche sur l'édition littéraire au Québec (GRELQ) de Sherbrooke. (J'y reviendrai dans une prochaine chronique, pour signaler l'accent mis depuis une dizaine d'années sur l'édition, particulièrement l'édition critique.) Le Guide donne enfin les principales sources concernant les associations, congrès et centres d'études québécoises et canadiennes à l'étranger. Comme on le constate, on y fait un bon tour du jardin.

\section{Études canadiennes: publications et thèses étrangères}

Il est intéressant de parcourir ensuite la compilation préparée par L. Jones. Cette publication bilingue, subventionnée par le ministère des Affaires extérieures et la Bibliothèque nationale du Canada est une mise à jour de Monographies et revues publiées à l'étranger dans le cadre des études canadiennes (1987). Après la rubrique inaugurale des bibliographies (toutes disciplines), viennent celles des divers champs d'études dont celui des «Langues et littératures» auquel je m'arrête brièvement.

Si l'on se fie à ce relevé, présenté comme incomplet et ne couvrant que les dix dernières années, le pays qui nous fait le plus bel accueil est l'Italie. Vingt-quatre titres sont répertoriés, la plupart étant des thèses de doctorat portant sur un éventail assez large d'auteurs et de textes: Aquin, Basile, Blais, Ducharme, Godbout (3), Guèvremont, Grandbois, Hémon, Lasnier, Morin (Paul), Nelligan (2), Ouellette (Fernand), Roy (Gabrielle), Savard, Thériault (Yves). Il faut ajouter à cela les ouvrages de L. Petroni (actes de colloque), de notre correspondante, la directrice du Centre d'études québécoises de Bologne, Franca Marcato (sur Ducharme: actuellement en traduction, son excellent ouvrage devrait paraître bientôt au Québec), et l'essai imposant de Paolo Carile, professeur à Ferrare, sur les relations de voyage et la Bibliothèque bleue: Lo Sguardo impedito, littéralement: «le regard entravé», celui de l'Européen sur le Nouveau Monde (qui fera naître les mythes du bon et du mauvais sauvage, du paradis terrestre et de l'horrible désert). Le concept de l'altérité qui oriente la réflexion de $\mathrm{P}$. Carile semble d'ailleurs avoir inspiré l'Altérité dans la littérature québécoise, recueil d'exposés d'un de ces séminaires annuels de Bagni 
di Lucca organisés par le Centre de Bologne sous le titre général de «La dérive des francophonies». Voilà qui dit assez l'importance de nos collègues italiens pour les études québécoises.

Aux États-Unis, qui viennent en deuxième place avec seize titres, les travaux scolaires sont beaucoup moins nombreux. Je relève tout de même une thèse qui compare la poésie canadienne-française à la poésie mexicoaméricaine. Il faut par ailleurs mentionner plusieurs ouvrages considérables. À part le numéro spécial de Yale French Studies (1983), je rappelle la publication de notre correspondant J. Weiss sur le French-Canadian Theatre (1986), ceux de P.G. Lewis: The Literary Vision of Gabrielle Roy: An Analysis of Her Works, (1984) et sur Traditionalism, Nationalism and Feminism: Women Writers of Québec (1985); encore sur G. Roy: l'analyse structurale de E. Reisman Babby, The Play of Language \& Spectacle (1985) et enfin celui de M. Cagnon sur The French Novel of Quebec (1986). Tous ces travaux témoignent à leur tour de la profondeur du regard états-unien sur les études québécoises.

La troisième place revient à la France, avec quatorze titres. Mais ce nombre donne à la France une importance actuellement contestable, si l'on se fie à ce repérage. Quelques thèses dont une en anglais sur le bilinguisme à Port-au-Port; quelques ouvrages publiés par des Canadiens (le Paul-Marie Lapointe, 1987, de R. Melançon; le Canada littéraire à la croisée des cultures, 1985, de S. Sarkany) et les livres bien connus de A. Viatte (Histoire comparée des littératures francophones, 1980) et de M. Ducrocq-Poirier (le Roman canadien de langue française de 1860 à 1958, paru en 1978). Voilà tout. On pourra s'interroger sur le sort des études québécoises en France, même du côté de l'enseignement comme Réginald Martel l'a récemment fait ${ }^{5}$. Mais il faudra tenir compte des oublis de l'inventaire de Mme Jones: le spécial «Recherches québécoises» de Littérature ( $\mathrm{n}^{\circ} 66,1987$ ) et sans doute plusieurs autres travaux universitaires dont ceux qu'animent $Y$. Resch depuis son Centre SaintLaurent d'Aix-en-Provence, C. Duchet et ses collègues de Paris VIII ou M. Ducrocq-Poirier à la Sorbonne, pour ne nommer que ceux-là. En fait, il nous faudrait un bilan plus précis pour la France, avant de sauter aux conclusions habituelles depuis la suppression des chaires québécoises.

En quatrième place vient l'Allemagne avec huit titres, renvoyant pour la plupart aux problématiques culturelle et linguistique, à l'exception des ouvrages de M. Larrass (Die Dichtung Alain Grandbois: eine franzözish-kanadische ars alchymica der Ich-Werdung, 1983) et de l'Ontarien P. Socken qui a publié à Francfort (Myth and Morality in Alexandre Chenevert by Gabrielle Roy, 1987). Prennent place ensuite l'Angleterre, grâce aux publications de C. May (Breaking the Silence: The Literature of Quebec, $2^{\mathrm{e}}$ éd. en 1985; Jacques Godbout «Salut Galarneau»: A Guide for Students and Teachers, 1984) et la Belgique avec trois publications de colloques (Trajectoires: littérature et institutions au Québec et en Belgique francophone, 1985; Littérature québécoise: voix d'un peuple, voies d'une autonomie, 1985; et (répertorié en sociologie) la Question sociale en Belgique et au Canada XIX ${ }^{\mathbf{e}} \mathbf{X X}^{\mathbf{e}}$ siècles, 
1988. Toutes ces publications trouvent leur origine au Centre d'études canadiennes de l'Université libre de Bruxelles, dirigé par G. Kurgan-Hentenrik. Ne sont pas compilés ici les travaux du Centre d'études québécoises de Liège où œuvrent J. Dubois et J.-M. Klinkenberg. Enfin, seule mention africaine, due à un collègue québécois, Max Dorsinville: le Pays natal: essais sur les littératures du Tiers-Monde et du Québec (1983).

Comme on le voit, cette compilation est davantage indicielle que probante. Il faudra que les bilans des pays étrangers soient plus sûrs pour autoriser une véritable analyse. Quant au Guide, son usage permettra sans doute de le parfaire au fur et à mesure des mises à jour, quoiqu'il soit à première vue tout à fait fiable. À suivre.

1 Nous accueillerons avec reconnaissance les renseignements utiles que l'on voudra bien nous faire parvenir à ce sujet (toutes activités: colloques, rapports et autres publications, formations de groupes, de revues, etc.).

2 Marcel Fortin, Yvan Lamonde et François Ricard, Guide de la littérature québécoise, Montréal, Boréal, 1988, 158 p.

3 Canadian Studies/Études canadlennes, Foreign Publications and Theses/ Publications et thèses canadiennes, compilation de Linda M. Jones, Ottawa, Conseil international des études canadiennes pour les Affaires extérieures du Canada, 1989, 175 p.

4 Ces questions viennent d'une analyse des sujets de thèses et mémoires que j'ai présentée au congrès de l'ALCQ a Winnipeg en 1986.

5 «L'enseignement de la littérature et de la civilisation québécoise ne veut plus dire grand-chose» [en France], la Presse, 6 mai 1989, p. K2. 